\title{
28 Research Square \\ Location-sharing Protocol for Privacy Protection in Mobile Online Social Networks
}

\section{Ou Ruan}

Hubei University of Technology

\section{Lixiao Zhang}

Hubei University of Technology

Yuanyuan Zhang ( $\sim$ circle0519@hotmail.com )

Hubei University of Technology

\section{Research}

Keywords: Location privacy, Location sharing, Mobile online social networks, Smart cities

Posted Date: January 15th, 2021

DOI: https://doi.org/10.21203/rs.3.rs-144558/v1

License: (1) This work is licensed under a Creative Commons Attribution 4.0 International License. Read Full License 


\section{Abstract}

Location-based services are becoming more and more popular in mobile online social networks(mOSNs) for smart cities, but users' privacy also has aroused wide concern, such as locations, friend sets and other private information. At present, many protocols have been proposed, but these protocols are inefficient and ignore some security risks. In the paper, we present a new location sharing protocol, which solves these two issues by using symmetric/asymmetric encryption properly. We adopt the following methods to reduce the communication and computation costs: only setting up one location server; connecting social network server and location server directly instead of through cellular towers; avoiding broadcast encryption. We introduce dummy identities to protect users' identity privacy, and prevent location

server from inferring users' activity tracks by updating dummy identities in time. The details of security and performance analysis with the related protocols show that our protocol enjoys two advantages: (1) it's more efficient than the related protocols, which greatly reduces the computation and communication costs; (2) it satisfies all security goals, however, most previous protocols only meet some security goals.

\section{Full Text}

This preprint is available for download as a PDF.

\section{Figures}
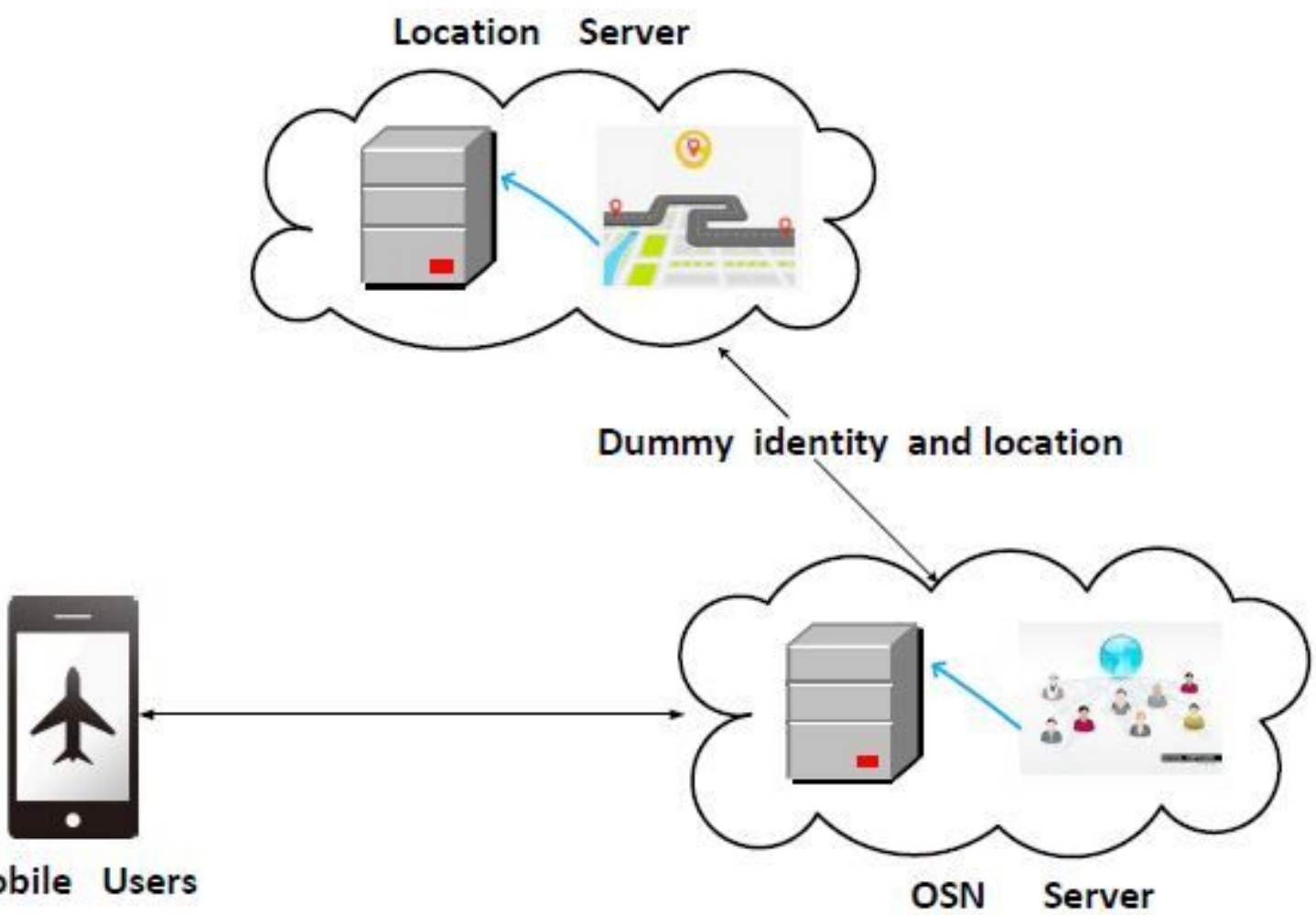
Figure 1

System architecture in mobile online social networks(mOSNs)

\begin{tabular}{ccc} 
Mobile User $\left(u_{i}\right)$ & Online social network Server $\left(S_{\text {OSN }}\right)$ & Location Server $\left(S_{L S}\right)$ \\
\hline$\left(p k_{I D_{i}}, s k_{D_{i}}\right)$ & $\begin{array}{l}\text { social network graph } G \\
\left(I D_{i}, d f_{I D}, d s_{D_{i}}, p k_{D_{i}}\right) \\
\left(p k_{o s n}, s k_{o s n}\right)\end{array}$ & $\left(p k_{L S}, s k_{L S}\right)$ \\
\hline
\end{tabular}

$$
\begin{aligned}
& \sigma_{I D_{i}}=\operatorname{Sig}_{s k_{M_{s}}}\left(I D_{i}, t s\right) \\
& c_{I D_{i}}=E_{p k_{m=x}}\left(I D_{i}, t s, \sigma_{D_{i}}\right) \\
& c=E_{p k_{L S}}(x, y) \quad \stackrel{\left(c_{m_{i}}, c\right)}{\longrightarrow}
\end{aligned}
$$$$
\begin{aligned}
& \left(I D_{i}, t s, \sigma_{I D_{i}}\right)=D_{s k_{m i}}\left(c_{I D}\right) \\
& \text { If }\left(\operatorname{Ver}_{p k_{N_{i}}}\left(\sigma_{I D_{i}}\right)=\left(I D_{i}, t s\right)\right)
\end{aligned}
$$

$S_{\text {OSN }}$ randomly generates a dummy identity $F I D_{i}$ and stores this updated record $\left(I D_{i}, F I D_{i}, d f_{I D_{i}}, d s_{I D_{i}}, p k_{\mathbb{D}_{i}}\right)$

\section{Figure 2}

User registration 


$$
\left(p k_{I I,}, s k_{I D_{i}}\right)
$$

social network graph $G$

$$
\begin{aligned}
& \left(I D_{i}, d f_{I D_{i}}, d s_{I I_{i}}, p k_{I D_{i}}\right) \\
& \left(p k_{o s s}, s k_{\text {oses }}\right)
\end{aligned}
$$

$$
\left(p k_{L s}, s k_{L S}\right)
$$

$$
\begin{aligned}
& \sigma_{I D_{i}}=\operatorname{Sig}_{s k_{\Phi_{i}}}\left(I D_{i}, t s\right) \\
& c=E_{p k_{t s}}(x, y) \\
& c_{I D_{i}}=E_{p k_{\text {and }}}\left(I D_{i}, t s, \sigma_{I D_{1}}\right)
\end{aligned}
$$

$\stackrel{\left(c_{I D Y}, c\right)}{\longrightarrow}$

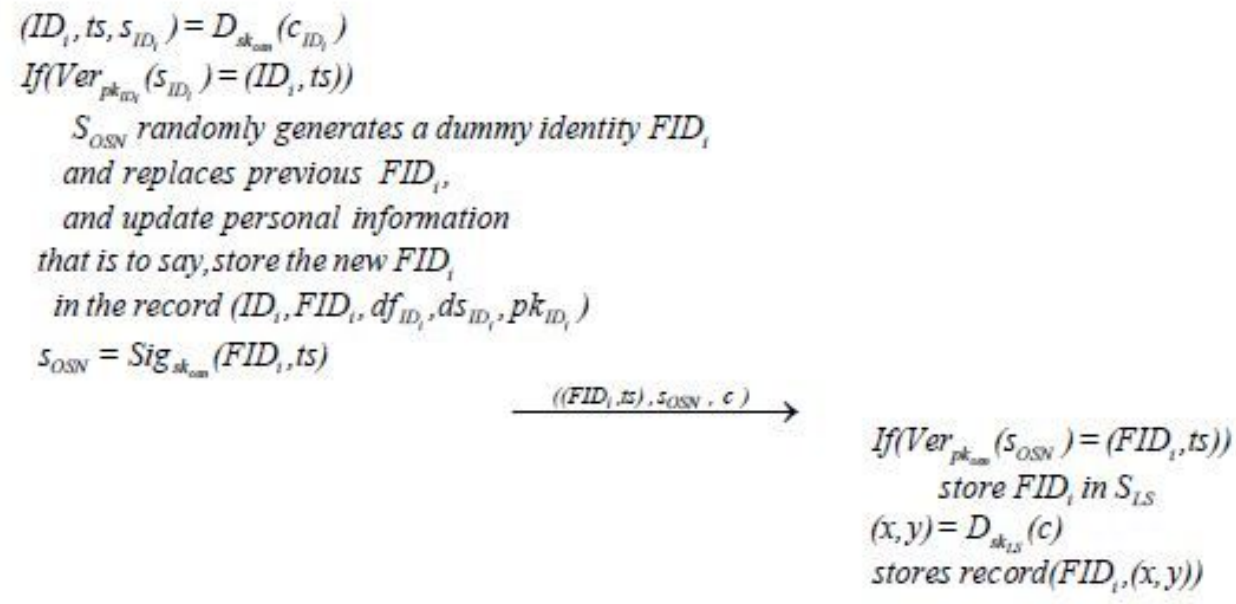

\section{Figure 3}

Location update 


$$
\left(p k_{I p_{1}}, s k_{l \underline{p}}\right)
$$

social network graph $G$

$$
\left(I D_{i}, d f_{I D_{1}}, d s_{I Q_{1}}, p k_{I D_{1}}\right)
$$$$
\left(p k_{\text {osn }}, s k_{\text {osn }}\right)
$$

$$
\left(p k_{L S}, s k_{L S}\right)
$$

The user $I D_{i}$ generates

$a$ one-time symmetric key $k$

$c=E_{p k_{L S}}(x, y, k)$

$c_{I D_{l}}=E_{p k_{\text {ise }}}\left(I D_{l}, F^{\prime}, l\right)$ $\stackrel{\left(c_{I D}, c\right)}{\longrightarrow}$

$\left(I D_{i}, F^{\prime}, l\right)=D_{s k_{n a g}}\left(c_{D,}\right)$

match $\left(I D_{j}, F I D_{j}, d f_{I D_{j}}\right)$ of every friend of $I D_{i}$

through social network graph $G$

compute $d m_{D D}$ of every friend, $d m_{D_{j}}=\min \left(d f_{W_{j}}, l\right)$

the set $S$ is made up of $\left(F I D_{j}, d m_{m D_{j}}\right.$ ) of all friends

suppose the number of $I D_{1}^{\prime} s$ friends is $m$

$S=\left\{\left(F I D_{1}, d m_{l D_{1}}\right),\left(F I D_{2}, d m_{l D_{2}}\right), \ldots\left(F I D_{m}, d m_{D_{w}}\right)\right\}$

$c_{L S}=E_{p \star_{i S}}(S)$

$$
\begin{aligned}
& \stackrel{M}{\operatorname{for}(j=1 ; j<=p ; j++)} \\
& \left(F I D_{f},\left(x_{j}, y_{j}\right)\right)=D_{k}\left(c_{m_{j}}\right) \\
& S_{I D_{l}}=\left(\left(F I D_{1},\left(x_{1}, y_{1}\right)\right), \ldots \ldots . .\left(F I D_{p},\left(x_{p}, y_{p}\right)\right)\right)
\end{aligned}
$$

$S=D_{s k_{i}}\left(c_{L S}\right)$

$(x, y, k)=D_{s k_{t s}}(c)$

for $(j=1 ; j<=m ; j++)$

match $\left(x_{f}, y_{j}\right)$ of every member in $S$

if $\operatorname{dist}\left((x, y),\left(x_{j}, y_{j}\right)\right)<=d m_{W_{j}}$

$c_{I D_{1}}=E_{k}\left(F I D_{j},\left(x_{j}, y_{j}\right)\right)$

the set $M$ is made up of $p$ eligible members $\stackrel{M}{M}^{M}=\left(c_{I D_{1}}, c_{I D_{2}}, c_{I D_{1}}, \ldots . . . c_{I D_{p}}\right)$

\section{Figure 4}

Friends' location query 


$$
\left(p k_{m_{2}}, s k_{m_{3}}\right)
$$

social network graph $G$

$\left(I D_{i}, d f_{I D_{l}}, d s_{M_{i}}, p k_{D_{i}}\right)$

$\left(p k_{\text {on }}, s k_{\text {om }}\right)$

$\left(p k_{L S}, s k_{L S}\right)$

The user ID generates

$a$ one-time symmetric key $k$

$c=E_{p k_{n}}(x, y, k)$

$c_{I D i}=E_{p k_{\text {sad }}}\left(I D_{i}, ' S ', l\right)$

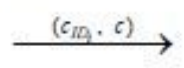

$\left(I_{i}, ' S^{\prime}, l\right)=D_{s k}\left(c_{m}\right)$

match $\left(I D_{j}, F D_{f}, d s_{I D}\right)$ of every stranger of $I D_{i}$

through social network graph $G$

compute $d m_{D D_{j}}$ of every stranger, $d m_{D_{j}}=\min \left(d s_{D_{j}}, l\right)$

the set $S$ is made up of $\left(F I D_{j}, d m_{I D_{j}}\right)$ of all strangers

suppose the number of ID's strangers is $m$

$S=\left\{\left(F I D_{1}, d m_{M D_{1}}\right),\left(F D_{2}, d m_{M D_{2}}\right), \ldots\left(F I D_{m}, d m_{I D_{n}}\right)\right\}$

$c_{L S}=E_{P k_{L S}}(S)$

$S=D_{s k_{z S}}\left(c_{L S}\right)$

$(x, y, k)=D_{s k_{1 x}}(c)$

for $(j=1 ; j<=m ; j++)$

match $\left(x_{j}, y_{j}\right)$ of every member in $S$

if $\operatorname{dist}\left((x, y),\left(x_{j}, y_{j}\right)\right)<=d m_{m}$

$$
c_{I D_{j}}=E_{k}\left(F I D_{j},\left(x_{j}, y_{j}\right)\right)
$$

the set $M$ is made up of $p$ eligible members

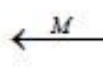

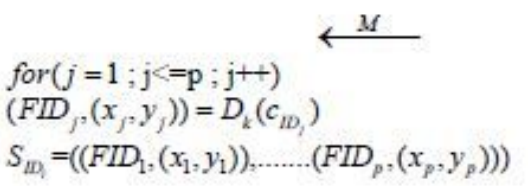

\section{Figure 5}

Strangers' location query 